\title{
SIEBECK CURVES AND TWO REFINEMENTS OF THE GAUSS-LUCAS THEOREM
}

\author{
EDUARDO CASAS-ALVERO*
}

\begin{abstract}
We prove two results which improve the well known Gauss-Lucas theorem by locating the roots of the derivative of a complex polynomial $f$ in sets smaller than the convex hull of the roots of $f$.
\end{abstract}

\section{Introduction}

Assume that $f \in \mathrm{C}[z]$ is a complex polynomial and let $z_{1}, \ldots, z_{m}$ be its distinct roots, viewed as points of the complex plane. The Gauss-Lucas theorem assures that the roots of $d f / d z$ belong to the convex hull of $z_{1}, \ldots, z_{m}$ (see for instance [10], 1.2.1). An old result due to J. Siebeck ([12], see also [10], 1.2.2) asserts that if $f$ has degree three and its roots are the vertices of a triangle $z_{1} z_{2} z_{3}$, then the roots of $d f / d z$ are the foci of the only conic $C$ tangent to the sides of $z_{1} z_{2} z_{3}$ at their midpoints. In his paper [7], B. Z. Linfield extended Siebeck's result to arbitrary polynomials: he proved that the roots of $d f / d z$ which are not roots of $f$ are the foci of an algebraic curve $\mathscr{S}$, of class $m-1$, determined by a set of tangential conditions (see 2.1 below; further details are in [4]). In the sequel the curve $\mathscr{S}$, which plays the role of the conic $C$ in Siebeck's result, will be called the Siebeck curve of $f$. In the same paper, Linfield stated the refinement of the Gauss-Lucas theorem described next.

For simplicity assume that the roots $z_{1}, \ldots, z_{m}$ of $f$ are simple and nonaligned (for the general claim see Theorem 7.1 below). Fix an arbitrary direction $w$ and, for $j=1, \ldots, m$, let $\ell_{j}$ be the line projecting $z_{j}$ in the direction $w$. Assume that $\ell_{j}$ has equation $a x+b y+c_{j}=0$, and take $g=: \prod_{j=1}^{m}\left(X-c_{j}\right) \in$ $\mathrm{R}[X]$. Let $c_{1}^{\prime}, \ldots, c_{m-1}^{\prime}$ be the roots of $d g / d X$. Consider the lines, also in the direction $w$,

$$
\ell_{j}^{\prime}: a x+b y+c_{j}^{\prime}=0, \quad j=1, \ldots, m-1 .
$$

By Rolle's theorem, they are real and each lies between two of the lines $\ell_{j}$. After a suitable reordering, assume that $\ell_{1}^{\prime}$ and $\ell_{m-1}^{\prime}$ are the extremal lines

\footnotetext{
* Partially supported by MTM-2009-14163-C02-01.

Received 25 January 2011, in final form 6 May 2011.
} 
among the $\ell_{i}^{\prime}$. Linfield's claim is that all the roots of $d f / d z$ which are not roots of $f$ lie in the open stripe $\Sigma_{w}$ bounded by $\ell_{1}^{\prime}$ and $\ell_{m-1}^{\prime}$. Then, by the arbitrariness of $w$, the roots of $d f / d z$ other than the roots of $f$ belong to the set $\Sigma:=\bigcap_{w} \Sigma_{w}$.

Let $w_{i}, \ldots, w_{r}$ be the directions of the sides of the convex hull $T$ of $z_{1}, \ldots, z_{m}$. Then $\Sigma_{w_{1}} \cap \cdots \cap \Sigma_{w_{r}}$ is the interior of $T$, as each side of $T$ appears at least twice among the lines $\ell_{j}$, and so it is one of the $\ell_{j}^{\prime}$. It follows that $\Sigma$ is included in the interior of $T$ and it is not difficult to see that in general this inclusion is strict (see 7.7). Therefore, Linfield's claim improves Gauss-Lucas' theorem.

Linfield's proof makes strong use of the Siebeck curve. Unfortunately it makes also use of a number of wrong claims about real plane curves. This may be probably the reason why no mention of Linfield's refinement seems to appear in posterior literature.

The purpose of this paper is to set a number of especial properties of the Siebeck curve and use them to make a correct proof of Linfield's result explained above. We will also obtain a second refinement of the Gauss-Lucas theorem, stronger than Linfield's one, but at the price of involving the Siebeck curve in its claim: the Siebeck curve $\mathscr{S}$ being bounded, we will prove that no root of $d f / d z$ other than the roots of $f$ lies in the closure of the unbounded connected component of the complementary of $\mathscr{S}$.

I'm indebted to C. D' Andrea, J. C. Naranjo, M. Sombra and G. Welters for fruitful conversations on this subject.

\section{Preliminaries}

Planes: To avoid any confusion, we will denote by $\mathrm{E}$ the field of the complex numbers $C$ viewed as a real Euclidean plane (with the metric induced by the complex absolute value). The projective closure of $\mathrm{E}$ will be denoted $\mathrm{P}, \Omega:=$ $P-E$ being its improper line. We will make intensive use of the dual plane $P^{\vee}$ of $\mathrm{P}$ : if $p^{*}$ denotes the pencil of lines through the point $p \in \mathrm{P}$, the points of $\mathrm{P}^{\vee}$ are the lines of $\mathrm{P}$ and its lines are the pencils $p^{*}, p \in \mathrm{P}$. Unless otherwise said we will take the real and imaginary part $x, y$ of any $z=x+y i \in \mathrm{C}$ as its affine coordinates in $\mathrm{E}$. The homogeneous coordinates (in $\mathrm{P}$ ) of the same point are $(1, x, y)$ and the homogeneous coordinates of a line $\ell: a x+b y+c=0$ (as a point of $\mathrm{P}^{\vee}$ ) are $(c, a, b)$. We will pass to the complex extensions of these planes by just allowing the coordinates to take complex values, and so we will deal with imaginary points and imaginary lines. In particular, the cyclic points of E are the imaginary improper points $I=[0,1, i]$ and $J=[0,1,-i]$.

Pencils of parallel lines $p^{*}, p \in \Omega$, will be always taken with their natural structure of affine line, in which the improper element is the improper line $\Omega$. 
GROUPS OF POINTS: A group of points (or effective divisor) of degree $r$ is a formal sum of $r$ points $\mathbf{G}=p_{1}+\cdots+p_{r}$. The multiplicity of $p_{j}$ in $\mathbf{G}$ is the number of times that $p_{j}$ appears in the sum. If the points $p_{j}$ belong to an affine line and have coordinates $a_{j}, j=1, \ldots, r$, then any equation $a\left(x-a_{1}\right) \ldots\left(x-a_{r}\right)=0, a \neq 0$, will be called an equation of $\mathbf{G}$.

Curves: Unless otherwise said, the word curve will mean plane algebraic curve, affine or projective. We will write $C: f=0$ to indicate that $C$ is the curve defined by the equation $f=0$. The degree of $f$ is the order (or degree ) of $C$. Curves will be allowed to have multiple components and the curve $C: f_{1} \ldots f_{r}=0$, composed of the curves $C_{j}: f_{j}=0, j=1, \ldots, r$, will be written $C=C_{1}+\cdots+C_{r}$. The intersection multiplicity of two curves $C$ and $C^{\prime}$ at a point $p$ will be denoted $\left[C \cdot C^{\prime}\right]_{p}$. If $C, C^{\prime}$ share no component, then $C \cdot C^{\prime}$ will denote the intersection group of $C, C^{\prime}$, namely the group of points

$$
C \cdot C^{\prime}=\sum_{p \in C \cap C^{\prime}}\left[C \cdot C^{\prime}\right]_{p} p .
$$

Envelopes: A line $\ell$ is taken as tangent to a curve $C$ at a point $p \in C$ if and only if the intersection multiplicity $[\ell \cdot C]_{p}$ is higher than the multiplicity of $C$ at $p$. Let $C$ be an irreducible curve of a real or complex projective plane $\mathrm{P}_{2}, \operatorname{deg} C>1$. The lines tangent to $C$ describe an irreducible curve $C^{*}$ of the dual plane $\mathrm{P}_{2}^{\vee}$, with $\operatorname{deg} C^{*}>1$, called the envelope of $C$. The map $C \mapsto C^{*}$ readily extends by linearity to a bijection between the sets of curves of $P_{2}$ and $\mathrm{P}_{2}^{\vee}$ containing no real or imaginary line as component. The image of a curve $C$ is still denoted $C^{*}$ and called the envelope of $C$, while $C$ itself is called the curve enveloped by $C^{*}$.

Augmented CuRves: In order to extend the above map $C \mapsto C^{*}$ to a bijection covering all curves of $\mathrm{P}_{2}^{\vee}$, we will consider augmented curves: an augmented curve of $\mathrm{P}_{2}$ is the formal sum of a group of points $\mathbf{G}=p_{1}+\cdots+p_{r}$ and a curve $C$, both of $\mathrm{P}_{2}$, noted $\mathscr{C}=\mathbf{G}+C$. We will refer to $\mathbf{G}$ and $C$ as the zero- and the one-dimensional part of $\mathscr{C}$, respectively. The points of either of these parts will be said to be the points of (or belonging to) $\mathscr{C}$. We will refer to the points of $\mathbf{G}$ as the nested points of $\mathscr{C}$, their multiplicities in $\mathbf{G}$ will be taken as their multiplicities in $\mathscr{C}$. Curves and groups of points will be taken as augmented curves with empty zero- and one-dimensional part, respectively. By definition, a line $\ell$ is tangent to the above augmented curve $\mathscr{C}$ at one of its points $p$ if and only if either $\ell$ is tangent to $C$ at $p$, or $p \in \mathbf{G}$ and $p \in \ell$. Consequently, we will take the envelope of $\mathscr{C}$ to be curve $\mathscr{C}^{*}=p_{1}^{*}+\cdots+p_{r}^{*}+C^{*}$ and it is direct to check that $\mathscr{C} \mapsto \mathscr{C}^{*}$ defines a bijection between augmented curves of $\mathrm{P}_{2}$ with no real or imaginary line in its one-dimensional part and the curves 
of $\mathrm{P}_{2}^{\vee}$. Then $\mathscr{C}$ will be called the augmented curve enveloped by $\mathscr{C}^{*}$. As for ordinary curves, the class of an augmented curve $\mathscr{C}$ is defined as being the order of its envelope $\mathscr{C}^{*}$ : if, as above, $\mathscr{C}=\mathbf{G}+C$, its class is the sum of the class of $C$ and the degree of $\mathbf{G}$.

FocI: We will restrict ourselves to consider (real) curves of $\mathrm{P}$ containing no real or imaginary lines and not tangent to $\Omega$. According to the classical definition (see [5], X.2), the foci of such a curve $C$ are the intersections of pairs of conjugate tangents to $C$ drawn from the cyclic points $I$ and $J$. Equivalently, a point $p \in \mathrm{E}$ is a focus of $C$ if and only if $p^{*}$, as a line of $\mathrm{P}^{\vee}$, joins two conjugate $\ell \in C^{*} \cap I^{*}$ and $\bar{\ell} \in C^{*} \cap J^{*}$. Note that $p$ is real; its multiplicity as a focus is defined to be

$$
\left[C^{*} \cdot I^{*}\right]_{\ell}=\left[C^{*} \cdot J^{*}\right]_{\bar{\ell}} .
$$

The foci of $C$, repeated according to multiplicities, compose the focal group of $C$. The degree of the focal group (the number of foci, if they are simple) is then the class of $C$. In this form, the above definitions apply without changes to the augmented curves $\mathscr{C}$ containing no real or imaginary line and non-tangent to $\Omega$. It is direct to check that the real nested points of $\mathscr{C}$ appear, with their multiplicities, as foci of $\mathscr{C}$.

Harmonic Group: Assume that $\mathrm{A}_{1}$ is a real affine line, and $Q=v_{1} q_{1}+$ $\cdots+v_{r} q_{r}$ a group of real points of $\mathrm{A}_{1}, q_{i} \neq q_{j}$ for $i \neq j$. The polar group $Q^{\prime}$ of $Q$ relative to the improper point is defined in the projective closure of $A_{1}$ as follows: if $q_{j}$ has affine coordinate $a_{j}, j=1, \ldots, r$, then $h=\prod_{j}\left(x-a_{j}\right)^{\nu_{j}}=0$ is an equation of $Q$ and $Q^{\prime}$ is the group of points whose coordinates are the zeros of $d h / d x$, repeated according multiplicities. It directly follows from elementary properties of the derivative that $\mathbf{H}(Q):=Q^{\prime}-\sum_{j}\left(v_{j}-1\right) q_{j}$ is a group of $r-1$ different points, all real and each placed between two consecutive points of $Q$. We will call $\mathbf{H}(Q)$ the harmonic group of $Q$. Its property of having one point between each two consecutive points of $Q$ will become fundamental in the sequel. We will refer to it as the separation property of the harmonic group. For instance the harmonic group of a group of two points $v_{1} q_{1}+v_{2} q_{2}$ has as its only point the point dividing the segment $q_{1} q_{2}$ in the ratio $v_{2} / v_{1}$, its midpoint if $v_{1}=v_{2}$.

SiebeCK CuRve: Assume as above that $f \in \mathrm{C}[z]$ has different roots $z_{1}, \ldots, z_{m} \in \mathrm{E}, m \geq 2$, and let $\mu_{j}$ be the multiplicity of $z_{j}, j=1, \ldots, m$. The pencils $z_{1}^{*}, \ldots, z_{m}^{*}$, of the lines through each of the roots $z_{j}$, are lines of $\mathrm{P}^{\vee}$; repeated according to the multiplicities of their corresponding roots, they compose the algebraic curve of $\mathrm{P}^{\vee} Z^{*}:=\mu_{1} z_{1}^{*}+\cdots+\mu_{m} z_{m}^{*}$. In other words, 
$Z^{*}$ is the envelope of the group of roots $Z=\mu_{1} z_{1}+\cdots+\mu_{m} z_{m}$ seen as an augmented curve without one-dimensional part. On the other hand, the improper line $\Omega$ of $\mathrm{P}$ is a distinguished point of $\mathrm{P}^{\vee}$. Since all the $z_{j}$ are proper points, $\Omega$ is not a point of $Z^{*}$ and we are allowed to consider the polar $\mathscr{P}_{\Omega}\left(Z^{*}\right)$ of $Z^{*}$ relative to the point $\Omega$. By an easy and well known property of the polars, each line $z_{j}^{*}$ appears as a component of multiplicity $\mu_{j}-1$ of $\mathscr{P}_{\Omega}\left(Z^{*}\right)$. After discarding from the polar these obvious components, we are left with

$$
\mathscr{S}^{*}:=\mathscr{P}_{\Omega}\left(Z^{*}\right)-\sum_{j=1}^{m}\left(\mu_{j}-1\right) z_{j}^{*},
$$

an algebraic curve in $\mathbf{P}^{\vee}$, of degree $m-1$. Following Linfield, we take the Siebeck curve of $f$ to be the augmented curve $\mathscr{S}$ of P enveloped by $\mathscr{S}^{*}$. We will refer to $\mathscr{S}^{*}$ as the Siebeck envelope of $f$. Using this definition, Linfield obtained the following general version of Siebeck's theorem, see [4], 6.1 and 7.3, for the proof:

THEOREM 2.1. If $f \in \mathrm{C}[z]$ has different roots $z_{1}, \ldots, z_{m}, m \geq 2$, with respective multiplicities $\mu_{1}, \ldots, \mu_{m}$, then

(1) The Siebeck curve of $f$ is the only augmented curve of class $m-1$ which, for each line $\ell$ joining two roots of $f$, is tangent to $\ell$ at the points of the harmonic group of the group of roots of $f$ lying on $\ell$.

(2) The foci of the Siebeck curve of $f$ agree, multiplicities included, with the roots of $d f / d z$ which are not roots of $f$.

The reader may see the example in Figure 1 below, and also the easier examples 6.8 and 7.8 in [4].

Actually, Linfield presented in [7] an even more general version dealing with rational functions. Although he was clear at the key point of defining the Siebeck curves, many of his proofs are obscure. The new proofs provided in [4] are for the polynomial case only.

We will make frequent use of the following two additional facts:

Proposition 2.2 ([4], 8.1). The group of tangents to $\mathscr{S}$ from an improper point $p$ is

$$
\mathscr{S}^{*} \cdot p^{*}=\mathbf{H}\left(Z^{*} \cdot p^{*}\right)+\sum_{\ell \in p^{*}, e_{\ell}>0}\left(e_{\ell}-1\right) \ell,
$$

where $\mathbf{H}$ denotes harmonic group (in $p^{*}$, taken as an affine line) and $e_{\ell}$ is the number of different roots of $f$ on $\ell$. 


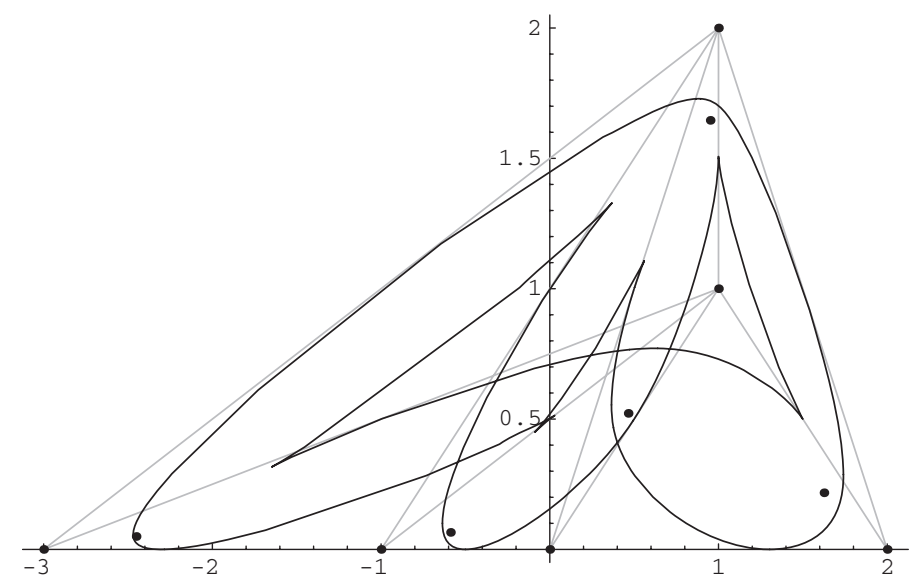

FIGURE 1. The roots of $f=z(z+1)(z-2)(z+3)(z-1-i)(z-1-2 i)$, the lines joining them, the corresponding Siebeck curve and the foci of the latter, which are the roots of $d f / d z$.

REMARK 2.3. Proposition 2.2 has a number of direct consequences, namely:

(1) $\Omega \notin \mathscr{S}^{*}$; in particular all nested points of $\mathscr{S}$ are proper.

(2) All the intersection points of $\mathscr{S}^{*}$ and $p^{*}$ are real. They all are simple, but for $p$ being the improper point of a line containing three or more roots of $f$.

(3) $\mathscr{S}^{*}$ has no imaginary or multiple component, which in particular applies to the lines contained in $\mathscr{S}^{*}$ showing that the nested points of $\mathscr{S}$ are real and have multiplicity one.

Proposition 2.4 ([4], 7.3, 7.7, 8.5). Each line $\ell$ of $\mathrm{E}$ containing $e \geq 2$ different roots of $f$ is an ordinary $(e-1)$-fold point (a smooth point if $e=2$ ) of $\mathscr{S}^{*}$ and the tangent cone to $\mathscr{S}^{*}$ at $\ell$ (up to identification by biduality) is the harmonic group of the group of roots of $f$ on $\ell . \mathscr{S}^{*}$ has no other real singularities.

REMARK 2.5. The tangent at a flex of an algebraic curve $C$ is a non-ordinary singularity of the envelope of $C$ (see [6], 5.4, for instance). Therefore, proposition 2.4 asserts in particular that (the one-dimensional part of) $\mathscr{S}$ has no flexes.

Although usually not mentioned in the old literature, there are Siebeck curves with non-empty zero-dimensional part, and so considering augmented curves cannot be avoided. For instance, if the roots of $f$ are simple and placed at the vertices of a parallelogram $\Pi$, then the corresponding Siebeck curve is composed of the ellipse tangent to the sides of $\Pi$ at their midpoints and the 
intersection point of the diagonals of $\Pi$. The reader may see also Examples 6.6 and 6.7 in [4]. We will study the zero-dimensional part of a Siebeck curve in Section 4. Next is a further example covering an exceptional and rather obvious case that will appear often in the sequel: we will refer to it as the collinear case.

EXAMPLE 2.6. Assume that all roots of $f$ belong to a line $\ell$. Then $\ell$ is a $\left(\sum_{j} \mu_{j}\right)$-fold point of $Z^{*}$ and so the polar $\mathscr{P}_{\Omega}\left(Z^{*}\right)$ has multiplicity at least $\sum_{j} \mu_{j}-1$ at $\ell$. Since, on the other hand, $\mathscr{P}_{\Omega}\left(Z^{*}\right)$ has degree $\sum_{j} \mu_{j}-1$, it is composed of lines of $\mathrm{P}^{\vee}$ through $\ell$ too, and therefore so is the Siebeck envelope $\mathscr{S}^{*}$. Consequently, the Siebeck curve consists of $m-1$ points and has no one-dimensional part. Furthermore, both $Z^{*}$ and $\mathscr{S}^{*}$ equal their respective tangent cones at $\ell$, after which, by 2.4 , the Siebeck curve equals the harmonic group, in $\ell$, of the group of roots of $f$.

\section{Variation of numbers of real points and real tangents}

In this section we will examine the local variation of the number of real intersections of a fixed algebraic curve and a variable line in a pencil, intersection points being always counted according to multiplicities. By duality, this will apply to the local variation of the number of real tangents to a fixed augmented curve from a point varying on a line. Before this, we state a fairly well known fact we will need in the sequel. For the convenience of the reader we sketch its proof.

Lemma 3.1. Assume that $C$ is an algebraic curve of a real projective plane $\mathrm{P}_{2}$ which contains no imaginary line. Then the set of (real) points of $\mathrm{P}_{2}$ from which there is an imaginary tangent to $C$ is open.

Proof. Since the imaginary tangents to $C$ remain unaffected after dropping real lines contained in $C$, we are allowed to assume that $C$ contains no real or imaginary line. Then the set of tangents to $C$ from a point $p \in \mathrm{P}_{2}$ is just $C^{*} \cap p^{*}$. Take projective coordinates in $\mathrm{P}_{2}$ and the corresponding dual coordinates in $\mathrm{P}_{2}^{\vee}$. Assume that there is an imaginary tangent from $p=\left[a_{0}, a_{1}, a_{2}\right]$ to $C$. Up to renumbering the coordinates we assume $a_{0}=1$, after which $p^{*}$ has equation, in dual coordinates, $u_{0}+a_{1} u_{1}+a_{2} u_{2}=0$. If $F\left(u_{0}, u_{1}, u_{2}\right)=0$ is an equation of $C^{*}$, by the hypothesis $F\left(-a_{1} u_{1}-a_{2} u_{2}, u_{1}, u_{2}\right)$ has a factor $u_{1}-b u_{2}$ with $b \in \mathrm{C}-\mathrm{R}$ and so $F\left(-a_{1} u-a_{2}, u, 1\right)$, as a polynomial in $u$, has the imaginary root $b$. By the continuity of the roots (see $[8]$, Th. $(1,4)$, for instance), for any $x_{1}, x_{2}$ close enough to $a_{1}, a_{2}, F\left(-x_{1} u-x_{2}, u, 1\right)$ has also an imaginary root and so $F\left(-x_{1} u_{1}-x_{2} u_{2}, u_{1}, u_{2}\right)$ has an imaginary linear factor. This in turn proves that from all points $\left[1, x_{1}, x_{2}\right]$ in a suitable neighbourhood of $p$ in $\mathrm{P}_{2}$, there is an imaginary tangent to $C$, as wanted. 
Remark 3.2. As the reader may check, the thesis of 3.1 does not hold for $C:\left(\left(x_{1}-2 x_{0}\right)^{2}+x_{2}^{2}-x_{0}^{2}\right)\left(x_{1}^{2}+x_{2}^{2}\right)=0$, which, indeed, contains imaginary lines.

Next we recall a few facts about the branches of a real curve at a point which will be used later on. Our considerations being local, assume that $C: h=0$, $h \in \mathrm{R}[x, y]$, is an affine algebraic curve, the affine coordinates $x, y$ being chosen so that the point $O \in C$ we are interested in is $O=(0,0)$. Assume that $h$ decomposes in irreducible factors

$$
h=u h_{1}^{\alpha_{1}} \ldots h_{r}^{\alpha_{r}},
$$

where $u, h_{1}, \ldots, h_{r} \in \mathrm{C}\{x, y\}, h_{j}(0,0)=0$ and $u$ is invertible. Then there is an open neighbourhood $U$ of $O$ so that each $h_{j}$ defines in $U$ an irreducible complex analytic curve $\gamma_{j}$, in such a way that two different $\gamma_{j}$ share no point other than $O$ and $C \cap U=\bigcup_{j} \gamma_{j}$. Each $\gamma_{j}$ (taken up to restriction to smaller neighbourhoods of $O$ ) is said to be a branch of $C$. The positive integer $\alpha_{j}$ is called the multiplicity of $\gamma_{j}$ (as a branch of $C$ ). Puiseux's theorem asserts that, for a suitable $U$ and each $j$, the points of $\gamma_{j}$ are given by a parameterization (Puiseux parameterization)

$$
x=t^{n_{j}}, \quad y=s_{j}(t)
$$

where $s_{j}(t) \in \mathrm{C}\{t\}$. We fix $j$ and write $s=s_{j}, n=n_{j}$ for simplicity. The intersection multiplicity of the branch $\gamma_{j}$ and a second curve $C^{\prime}: h^{\prime}=0$ is

$$
\left[\gamma_{j} \cdot C^{\prime}\right]=\operatorname{ord}_{t} h^{\prime}\left(t^{n}, s(t)\right) .
$$

If $n^{\prime}=\operatorname{ord}_{t} s(t)$, it follows in particular that for all lines $\ell$ through $O$ but a single one, it is

$$
\left[\gamma_{j} \cdot \ell\right]=\min \left\{n, n^{\prime}\right\}
$$

which is called the order of $\gamma_{j}$. The line $\ell^{\prime}$ excepted above is, by definition, a tangent to $C$ at $O$, usually called the tangent to $\gamma_{j}$; the difference

$$
\left[\gamma_{j} \cdot \ell^{\prime}\right]-\min \left\{n, n^{\prime}\right\}
$$

is called the class of the branch $\gamma_{j}$. In particular, if $n>n^{\prime}$, the tangent line is $x=0$ and $\gamma_{j}$ has order $n^{\prime}$ and class $n-n^{\prime}$; in case $n<n^{\prime}$, the tangent line is $y=0$ and $\gamma_{j}$ has order $n$ and class $n^{\prime}-n$, while for $n=n^{\prime}$ neither of the coordinate axes is tangent to $\gamma_{j}$.

The branch $\gamma_{j}$ is called imaginary if and only if it has no real points other than $O$ in a neighbourhood of $O$. Otherwise it is called real. In the first case it is clear that $s_{j}(t) \notin \mathrm{R}\{t\}$, while in the second one an easy argument (see [9], 
Lemma 3.3 and the note following it) shows that, up to reversing the orientation of the first axis, the series $s(t)$ may be taken in $\mathrm{R}\{t\}$.

Proposition 3.3. Assume that $C$ is an algebraic curve and $p^{*}$ a pencil of lines of a real projective plane $P_{2}$. Fix an absolute coordinate $\lambda$ in $p^{*}$ and write $\ell_{\lambda}$ the line of $p^{*}$ with absolute coordinate $\lambda$. Assume that $\ell_{0}$ is not tangent to $C$ at $p$. For any positive and small enough real number $\varepsilon$ :

(1) The number of real intersections of $C$ and $\ell_{\lambda}$ is independent of $\lambda$ for $-\varepsilon<\lambda<0$, and also for $0<\lambda<\varepsilon$. Denote these numbers by $\nu_{-}$and $\nu_{+}$, respectively, and by $v_{0}$ the number of real intersections of $C$ and $\ell_{0}$.

(2) $v_{0} \geq \max \left\{v_{-}, v_{+}\right\}$.

(3) $v_{-}=v_{+}$unless either

(a) there is an even order real branch of $C$ which is not tangent to $\ell_{0}$ and whose origin belongs to $\ell_{0}$ and is not $p$, or

(b) there is a real branch of $C$ which is tangent to $\ell_{0}$ and whose order and class have the same parity.

Proof. Since $\ell_{0}$ is assumed not to be tangent to $C$ at $p$, neither is any of the $\ell_{\lambda}$ for $|\lambda|$ small enough, and therefore the number of times $p$ has to be counted in the intersection of $C$ and $\ell_{\lambda}$ is independent of $\lambda$. Hence, to prove the claim it will be enough to consider the numbers of real intersections other than $p$.

Fix an affine chart of $\mathrm{P}_{2}$ and affine coordinates $x, y$ on it in such a way that $p$ is the improper point on the $y$-axis and the line $\ell_{\lambda}$ has equation $x=\lambda$, $\lambda \in \mathrm{R}$. In particular $\ell_{0}$ is the $y$-axis. By the above, we will reduce ourselves to counting the intersections lying in the affine chart. Let $h \in \mathrm{R}[x, y]$ be an equation of the affine part of $C$. If $d=\operatorname{deg}_{y} h$, there is a non-zero monomial $a_{0, d} y^{d}$ in $h$, as otherwise $C$ would be tangent to $\ell_{0}$ at $p$. This assures (see for instance [3], ex. 1.9) that there is $\varepsilon \in \mathrm{R}^{+}$for which all points $(x, y)$ of $C$ with $0<|x|<\varepsilon$ belong to one and only one of the branches of $C$ with origin at a point of $C$ on the $y$-axis. We may thus make our counts branch by branch and just add-up afterwards. Fix a branch $\gamma$ of $C$ with origin at a (real) point $O$ on the $y$-axis.

If $\gamma$ is imaginary, then it has no real intersection with $\ell_{\lambda}$ for $\lambda \neq 0$.

If, otherwise, $\gamma$ is real, then, after moving the origin, shrinking $\varepsilon$ and taking $-x$ instead of $x$ (and therefore $-\lambda$ instead of $\lambda$ ) if needed, we may assume without restriction that $O=(0,0)$ and the points of $\gamma$ are given by a Puiseux parameterization

$$
x=t^{n}, \quad y=t^{n^{\prime}} u(t)
$$

with $u(t) \in \mathrm{R}\{t\}, u(0) \neq 0$. On one hand it is clear that $O$ is the only intersection point of $\gamma$ and $\ell_{0}: x=0$, their intersection multiplicity at $O$ being $n$. 
On the other, for $\lambda \neq 0$ small enough, $\gamma$ and $\ell_{\lambda}: x-\lambda=0$ have $\mathrm{n}$ different complex intersections, all of them simple. A single one of these intersections is real if $n$ is odd, while for $n$ even there are two real intersections for $\lambda>0$ and no one for $\lambda<0$.

The above clearly proves claims (1) and (2) and shows that claim (3) is satisfied if all branches are either imaginary or real with $n$ odd. Assume that there is a real branch $\gamma$ as above with $n$ even. If $n \leq n^{\prime}$, then $\gamma$ has order $n$ and is not tangent to the $y$-axis (i.e., to $\ell_{0}$ ), which is the case excluded in (a). If still $n$ is even and $n>n^{\prime}$, then $\gamma$ is tangent to the $y$-axis, has order $n^{\prime}$ and class $n-n^{\prime}$, which has been excluded in (b).

Proposition 3.4. Assume that $C$ is an algebraic curve containing no line and $\ell$ a line of a real projective plane $\mathrm{P}_{2}$. Fix an absolute coordinate $\lambda$ in $\ell$ and write $p_{\lambda}$ the point of $\ell$ with absolute coordinate $\lambda$. Assume that $\ell$ is not tangent to $C$ at $p_{0}$. For any positive and small enough real number $\varepsilon$ :

(1) The number of real tangents to $C$ from $p_{\lambda}$ is independent of $\lambda$ for $-\varepsilon<$ $\lambda<0$, and also for $0<\lambda<\varepsilon$. Denote these numbers by $\tau_{-}$and $\tau_{+}$, respectively, and by $\tau_{0}$ the number of real tangents to $C$ from $p_{0}$.

(2) $\tau_{0} \geq \max \left\{\tau_{-}, \tau_{+}\right\}$.

(3) $\tau_{-}=\tau_{+}$unless either

(a) there is an even class real branch of $C$ which has not origin $p_{0}$ and whose tangent goes through $p_{0}$ and is not $\ell$, or

(b) there is a real branch of $C$ which has origin $p_{0}$ and whose order and class have the same parity.

Proof. Since $C$ contains no line, there is a one to one correspondence $\gamma \mapsto \gamma^{*}$, between the sets of branches of $C$ and its envelope $C^{*}$, so that if $\gamma$ has origin $p$, tangent $T$, order $n$ and class $\bar{n}$, then $\gamma^{*}$ has origin $T$, tangent $p^{*}$, order $\bar{n}$ and class $n$ (see for instance [13] or [6]). Furthermore, by definition, the number of times a line $\ell$ is counted among the tangents to $C$ through a point $p$ is the multiplicity of intersection $\left[p^{*} \cdot C^{*}\right]_{\ell}$. After this, it is enough to apply 3.3 to the envelope $C^{*}$.

REMARK 3.5. As the reader may check, the numbers of tangents to the cubic $y=x^{3}$ from points above and below the $x$-axis are different, which shows that the claim 3.4(3) may fail in the excepted case (a). Regarding the need of exception (b), just consider a smooth conic and let the point move on a line transverse to it. 


\section{The zero-dimensional part of $\mathscr{S}$}

In this section we characterize the nested points of $\mathscr{S}$ and prove that, the collinear case excepted, a Siebeck curve has at most a single nested point. We already know from 2.3 that the nested points of $\mathscr{S}$ are real, proper and have multiplicity one. The characterization of the nested points is as follows:

Proposition 4.1. A point $p \in \mathrm{E}$ is a nested point of the Siebeck curve $\mathscr{S}$ of a polynomial $f$ if and only if there are lines $\ell_{1}, \ldots, \ell_{r}$ through $p$ such that:

(1) Each root of $f$ belongs to one of the lines $\ell_{j}$ and each $\ell_{j}$ contains at least two different roots of $f$.

(2) For each $j=1, \ldots, r, p$ belongs to the harmonic group (in $\ell_{j}$ ) of the group of the roots of $f$ lying on $\ell_{j}$, taken with their multiplicities as roots.

Proof. Let $p$ be a nested point. Then the line $p^{*}$ of $\mathrm{P}^{\vee}$ is part of the polar $\mathscr{P}_{\Omega}\left(Z^{*}\right)$ and therefore, by a well known property of the polar curves, its intersections with $Z^{*}$ are either contact points of tangents to $Z^{*}$ from $\Omega$, or singular points of $Z^{*}$. Since $Z^{*}$ is composed of lines missing $\Omega$, the first case does not occur and so all the intersections of $p^{*}$ and $Z^{*}$ are points of $\mathrm{P}^{\vee}$ common to at least two components of $Z^{*}$; equivalently, the lines of $\mathrm{P}$ through $p$ and one of the roots of $f$ contain at least another root. Take $\ell_{1}, \ldots, \ell_{r}$ to be these lines; then condition (1) of the claim is clearly satisfied and condition (2) follows from 2.4.

For the converse, assume that the conditions of the claim are satisfied and let $e_{j}$ be the number of distinct roots of $f$ on $\ell_{j}, e_{j} \geq 2$ by the hypothesis. Let us pay some attention to the intersection multiplicities of $p^{*}$ and $\mathscr{S}^{*}$ at each $\ell_{j}$. By $2.4, p^{*}$ is part of the tangent cone to $\mathscr{S}^{*}$ at $\ell_{j}$ and so its intersection multiplicity with $\mathscr{S}^{*}$ at $\ell_{j}$ is strictly higher than the multiplicity of $\mathscr{S}^{*}$ at $\ell_{j}$, which by 2.4 is $e_{j}-1$. In other words,

$$
\left[p^{*} \cdot \mathscr{S}^{*}\right]_{\ell_{j}} \geq e_{j} .
$$

By condition (2) $p$ is not a root of $f$ and therefore no two different $\ell_{j}$ share a root; so adding up te above inequalities gives

$$
\sum_{j=1}^{r}\left[p^{*} \cdot \mathscr{S}^{*}\right]_{\ell_{j}} \geq \sum_{j=1}^{r} e_{j}=m .
$$

Bezout's theorem forces then $p^{*} \subset \mathscr{S}^{*}$ as wanted.

The reader may check the cases of nested points already mentioned in Section 2. 
REMARK 4.2. If no three different roots of $f$ are aligned, the conditions of 4.1 are satisfied if and only if the roots of $f$ may be arranged in disjoint unordered pairs of distinct roots in such a way that for each pair, say $\left\{z_{j}, z_{r}\right\}$, $p$ belongs to the segment $z_{j} z_{r}$ and divides it in the ratio $\mu_{r} / \mu_{j}$. In particular, in this case, the number of roots $m$ needs to be even and the nested point is obviously unique, as it is the center of gravity of the roots weighted according to multiplicities.

Nested points may also appear when the number of roots is odd; then, by 4.2 , there should be at least three aligned roots. Next is an example that may be directly checked using 4.1 .

EXAMPLE 4.3. If the polynomial $f$ has roots $-2,-1,2 / 3, i,-i$, all simple, then its Siebeck curve has 0 as nested point.

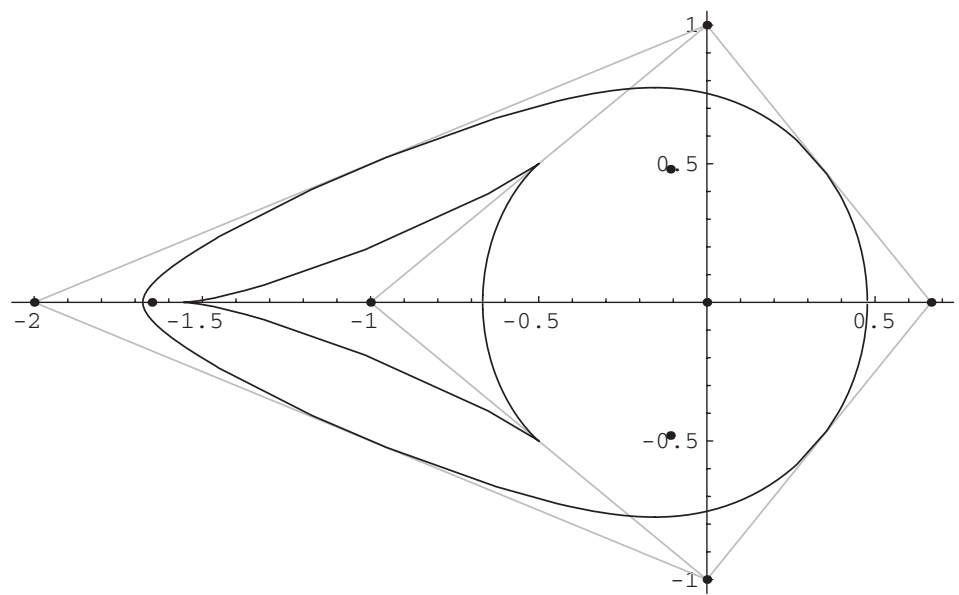

FIGURE 2. The Siebeck curve of Example 4.3. The origin is a nested point.

COROLlary 4.4. With the notations being as in 4.1, assume that $\mathscr{S}$ has a nested point $p$ and that, up to renumbering, the roots of $f$ lying on the line $\ell_{j}$ are $z_{1}, \ldots, z_{e}, 2 \leq e<m$. If $\bar{f}=f /\left(z-z_{1}\right)^{\mu_{1}} \ldots\left(z-z_{e}\right)^{\mu_{e}}$, then $\bar{f}$ has at least two roots and the Siebeck curve of $\bar{f}$ also has $p$ as nested point.

Proof. Direct from 4.1, as the conditions on the other roots remain the same after dropping $z_{1}, \ldots, z_{e}$.

The uniqueness of the nested point holds in all cases but in the collinear one, namely:

Proposition 4.5. If $f$ has three non-aligned roots, then its Siebeck curve has at most one nested point. 
Proof. Assume that $p$ and $p^{\prime}$ are different nested points of $\mathscr{S}$. Then the line $\ell$ joining them is a multiple tangent to $\mathscr{S}$ and therefore, by 2.4 , contains at least three different roots of $f$. Assume that the roots of $f$ on $\ell$ are $z_{1}, \ldots, z_{e}$, $e \geq 3$, and take $\bar{f}=f /\left(z-z_{1}\right)^{\mu_{1}} \ldots\left(z-z_{e}\right)^{\mu_{e}}$. Since $e<m$ due to the hypothesis of non-alignement of the roots, 4.4 applies and assures that both $p$ and $p^{\prime}$ are nested points of the Siebeck curve of $\bar{f}$. Then still $\ell$ is a multiple tangent to the Siebeck curve of $\bar{f}$, against the fact that no root of $\bar{f}$ lies on $\ell$.

COROLlary 4.6. The Siebeck curve has no one-dimensional part if and only if the roots of $f$ are aligned.

Proof. The if part has been seen in Example 2.6. For the converse, the nested points having multiplicity one, if $\mathscr{S}$ has a single nested point and no onedimensional part, then $m=2$ and the claim is obviously satisfied. Otherwise there are at least two nested points and 4.5 applies.

PROPOSITION 4.7. If not all roots of $f$ are collinear and $\mathscr{S}$ has nested point $p$, then there are imaginary tangents from $p$ to the one-dimensional part of $\mathscr{S}$.

Proof. Denote by $\tilde{\mathscr{S}}$ the one-dimensional part of $\mathscr{S}$ and assume that $\ell$ is a real tangent to $\tilde{\mathscr{S}}$ from $p$. Then $\ell$ is a multiple tangent to $\mathscr{S}$ and therefore (2.4) a line joining $e \geq 3$ roots of $f$ : it is thus one of the lines $\ell_{j}$ of 4.1 with $e_{j} \geq 3$. Conversely, any $\ell_{j}$ with $e_{j} \geq 3$ is, by 2.4 , an $\left(e_{j}-1\right)$-fold tangent to $\mathscr{S}$ and hence, the nested point being simple, a $\left(e_{j}-2\right)$-fold tangent to $\tilde{\mathscr{S}}$. The number of real tangents from $p$ to $\tilde{\mathscr{S}}$ (counted with multiplicities) is thus

$$
\sum_{j=1}^{r}\left(e_{j}-2\right),
$$

the terms with $e_{j}=2$ being of course irrelevant. As above, $\sum_{j=1}^{r} e_{j}=m$ and so the number of real tangents is $m-2 r$, strictly less than the class $m-2$ of $\tilde{\mathscr{S}}$ except for $r=1$, which clearly is the collinear case.

\section{The topology of $\mathscr{S}^{*}$}

In this section we will describe the topology of the Siebeck envelope $\mathscr{S}^{*}$ in order to obtain some results relative to numbers of real tangents. The usual results on the topology of real curves (such as those in [2]) being for the nonsingular case, they do not apply here and so we will proceed directly. Along the whole section we will be placed on the dual plane $\mathrm{P}^{\vee}$ and so, unless otherwise stated, the words point and line will mean point and line of $\mathrm{P}^{\vee}$.

As before we fix $f \in \mathrm{C}[z]$, with $m$ different roots, $z_{1}, \ldots, z_{m}, m \geq 2$, the multiplicity of $z_{j}$ being $\mu_{j}$. Since $z_{1}, \ldots, z_{m}$ are proper points of $\mathrm{P}, \Omega \notin z_{j}^{*}$, 
$j=1, \ldots, m$, and we may take in $\mathrm{P}^{\vee}$ homogeneous coordinates $u_{0}, u_{1}, u_{2}$ so that the third vertex of the reference is $[0,0,1]=\Omega$ and the side $u_{0}=0$ contains no intersection point of two different $z_{j}^{*}$. We will consider the affine chart $u_{0} \neq 0$ as an affine plane A with coordinates $X=u_{1} / u_{0}$ and $Y=u_{2} / u_{0}$ and we conventionally assume that the $X$-axis and the $Y$-axis are, respectively, horizontal, oriented from left to right, and vertical, oriented from bottom to top. The line $\Lambda_{\infty}: u_{0}=0$ is then the improper line of $\mathrm{A}$ and we assign to each of its points $\left[0, u_{1}, u_{2}\right]$ absolute coordinate $M=u_{2} / u_{1}$; in this way the point $\Omega$ has coordinate $1 / 0=\infty$ and the lines of $A$ with slope $M$ have as improper point the point with absolute coordinate $M$.

Consider again the curve $Z^{*}=\mu_{1} z_{1}^{*}+\cdots+\mu_{m} z_{m}^{*}$ of $\mathrm{P}^{\vee}$. As noted above, $\Omega \notin z_{j}^{*}, j=1, \ldots, m$, and so the lines $z_{j}^{*}$ are proper and non-vertical. Let $\bar{\alpha}_{j}$ be the absolute coordinate of the improper point (or, equivalently, the slope) of $z_{j}^{*}$, All these coordinates are finite and our choice of the coordinate frame assures that they are distinct. Thus, up to renumbering the roots of $f$, we will assume in the sequel that

$$
\bar{\alpha}_{1}>\cdots>\bar{\alpha}_{m} .
$$

For each $x \in \mathrm{R}$, let $\Lambda_{x}$ be the vertical line whose points have abscissa $x$. For each $q \in \Lambda_{x}$, let $e_{q}$ be the number of distinct lines $z_{j}^{*}$ through $q$. Denote by

$$
\alpha_{1}(x), \ldots, \alpha_{m}(x)
$$

the ordinates of the points $q \in \Lambda_{x}$ lying in one of the lines $z_{1}^{*}, \ldots, z_{m}^{*}$, each repeated $e_{q}$ times and the whole of them numbered so that

$$
\alpha_{1}(x) \geq \cdots \geq \alpha_{m}(x) .
$$

Note that there is no reason for having $\left(x, \alpha_{j}(x)\right) \in z_{j}^{*}$.

The definition of the Siebeck envelope $\mathscr{S}^{*}$ of $f$ was

$$
\mathscr{S}^{*}=\mathscr{P}_{\Omega}\left(Z^{*}\right)-\left(\mu_{1}-1\right) z_{1}^{*}-\cdots-\left(\mu_{m}-1\right) z_{m}^{*},
$$

$\mathscr{P}_{\Omega}$ meaning polar relative to $\Omega$. In particular $\mathscr{S}^{*}$ has degree $m-1$ and $\Omega \notin \mathscr{S}^{*}$, by 2.3 . Then, for all $x \in \mathrm{R}$, the (maybe imaginary) intersection points of $\Lambda_{x}$ and $\mathscr{S}^{*}$ belong to A: let $\beta_{1}(x), \ldots, \beta_{m-1}(x)$ be their ordinates, each repeated according to the corresponding intersection multiplicity. Assume that the improper points of $S^{*}$ are $q_{1}, \ldots, q_{m-1}$, and have absolute coordinates $\bar{\beta}_{1}, \ldots, \bar{\beta}_{m-1}$, respectively. By 2.2 , the intersection group of $\mathscr{S}^{*}$ and $\Lambda_{x}$, the case $x=\infty$ included, is

$$
\mathscr{S}^{*} \cdot \Lambda_{x}=\mathbf{H}\left(Z^{*} \cdot \Lambda_{x}\right)+\sum_{q \in \Lambda_{x}, e_{q}>0}\left(e_{q}-1\right) q
$$


Then the separation property of the harmonic group directly gives:

Lemma 5.1. (a) The numbers $\beta_{j}(x)$ are all real and may be numbered so that

$$
\alpha_{1}(x) \geq \beta_{1}(x) \geq \alpha_{2}(x) \geq \cdots \geq \alpha_{m-1}(x) \geq \beta_{m-1}(x) \geq \alpha_{m}(x) .
$$

Furthermore, an equality $\alpha_{j-1}(x)=\beta_{j-1}(x)$ or $\beta_{j-1}(x)=\alpha_{j}(x)$ holds if and only if $\alpha_{j-1}(x)=\alpha_{j}(x)$.

(b) The absolute coordinates $\bar{\beta}_{1}, \ldots, \bar{\beta}_{m-1}$ are all real and may be numbered so that

$$
\bar{\alpha}_{1}>\bar{\beta}_{1}>\bar{\alpha}_{2}>\cdots>\bar{\alpha}_{m-1}>\bar{\beta}_{m-1}>\bar{\alpha}_{m} .
$$

From now on, the real numbers $\beta_{1}(x), \ldots, \beta_{m-1}(x)$ and $\bar{\beta}_{1}, \ldots, \bar{\beta}_{m-i}$ will be assumed to be numbered as allowed by 5.1. By the continuity of the algebraic functions (see for instance [3], Ex. 1.9), each $\beta_{j}(x)$ is a continuous function and we may consider the continuous curves

$$
\delta_{j}=\left\{\left(x, \beta_{j}(x)\right) \mid x \in \mathrm{R}\right\}, \quad j=1, \ldots, m-1 .
$$

all contained in $\mathscr{S}^{*}$.

For each $j$, the closure $\bar{\delta}_{j}$ of $\delta_{j}$ in $\mathrm{P}^{\vee}$ is obtained by adding to it improper points of $\mathscr{S}^{*}$, precisely those which have absolute coordinates $\lim _{x \rightarrow+\infty} \beta_{j}(x) / x$ and $\lim _{x \rightarrow-\infty} \beta_{j}(x) / x$. In order to handle them, we need:

Lemma 5.2. For $j=1, \ldots, m$, it holds

$$
\lim _{x \rightarrow+\infty} \frac{\alpha_{j}(x)}{x}=\bar{\alpha}_{j} \quad \text { and } \quad \lim _{x \rightarrow-\infty} \frac{\alpha_{j}(x)}{x}=\bar{\alpha}_{m-j+1}
$$

Proof. For a fixed $j$ and any $x$ close enough to $+\infty$, all the points $\left(x, \alpha_{j}(x)\right)$ belong to one of the lines $z_{r}^{*}$, and each of these lines has a single point $\left(x, \alpha_{j}(x)\right)$ belonging to it. Therefore

$$
\left\{\lim _{x \rightarrow+\infty} \frac{\alpha_{j}(x)}{x}\right\}_{j=1, \ldots, m}=\left\{\bar{\alpha}_{1}, \ldots, \bar{\alpha}_{m}\right\} .
$$

Furthermore, the inequalities (5) give

$$
\lim _{x \rightarrow+\infty} \frac{\alpha_{1}(x)}{x} \geq \cdots \geq \lim _{x \rightarrow+\infty} \frac{\alpha_{m}(x)}{x},
$$


which, compared with the inequalities (1), prove the equalities on the left. The same argument, this time being

$$
\lim _{x \rightarrow-\infty} \frac{\alpha_{1}(x)}{x} \leq \cdots \leq \lim _{x \rightarrow-\infty} \frac{\alpha_{m}(x)}{x}
$$

proves the equalities on the right.

Regarding the $\beta_{j}(x)$ we have:

LEMMA 5.3. For $j=1, \ldots, m-1$,

$$
\lim _{x \rightarrow+\infty} \frac{\beta_{j}(x)}{x}=\bar{\beta}_{j} \quad \text { and } \quad \lim _{x \rightarrow-\infty} \frac{\beta_{j}(x)}{x}=\bar{\beta}_{m-j}
$$

Proof. As noted above, $\lim _{x \rightarrow+\infty} \beta_{j}(x) / x$ is one of the $\bar{\beta}_{r}$. On the other hand, the inequalities (3) together with Lemma 5.2 give

$$
\bar{\alpha}_{j} \geq \lim _{x \rightarrow+\infty} \frac{\beta_{j}(x)}{x} \geq \bar{\alpha}_{j+1} .
$$

Since the inequalities (4) assure that no $\bar{\beta}_{r}$ other than $\bar{\beta}_{j}$ belongs to $\left[\bar{\alpha}_{j+1}, \bar{\alpha}_{j}\right]$, the first group of equalities follows. A similar argument proves the second one.

We have thus $m-1 \operatorname{arcs} \bar{\delta}_{1}, \ldots, \bar{\delta}_{m-1}$ in $\mathrm{P}^{\vee}$, parameterized by $x \in[-\infty$, $+\infty]$, the endpoints of each $\bar{\delta}_{j}$ being the improper points $q_{m-j}$ and $q_{j}$. By 5.1 , no proper point of $\bar{\delta}_{j-1}$ is placed below the point of $\bar{\delta}_{j}$ with same $x$, for $j=2, \ldots, m-1$, and the absolute coordinates of the improper points $q_{1}, \ldots, q_{m-1}$, taken in this order, compose a strictly decreasing sequence. It is also clear that the $\operatorname{arcs} \bar{\delta}_{1}, \ldots, \bar{\delta}_{m-1}$ cover the set of real points of $\mathscr{S}^{*}$; their intersection points are determined next.

LEMMA 5.4. For $e \geq 3$, a point $p \in \mathrm{P}^{\vee}$ belongs to exactly $e-1$ (necessarily consecutive) of the arcs $\bar{\delta}_{j}$ if and only if exactly e lines among $z_{1}^{*}, \ldots, z_{m}^{*}$ are going through $p$.

Proof. Direct from 5.1 and the inequalities (4).

The points $p$ in the conditions of 5.4 are the lines of $\mathrm{P}$ containing $e$ different roots of $f$, already described as points of $\mathscr{S}^{*}$ in 2.4. We know in particular that all branches of $\mathscr{S}^{*}$ at its singular points have order one and no one has vertical tangent. It follows that their Puiseux parameterizations may be taken of the form $X=a+t, Y=s(t)$, where $a$ is the abscissa of the origin of the branch and $s \in \mathrm{R}\{t\}$. The parts of such a branch defined by the inequalities $t \leq 0$ and $t \geq 0$ will be called its half-branches. 
REMARK 5.5. Locally at any of the (e-1)-fold points $p$ of $\mathscr{S}^{*}, e \geq 3$, each $\operatorname{arc} \bar{\delta}_{j}$ is the union of two half-branches of $\mathscr{S}^{*}$ which are not necessarily part of the same branch. For instance, locally at $p$, from the $\operatorname{arcs} \bar{\delta}_{j}$ through $p$, the one with minimal $j$ is composed of one half of each of the two branches of $\mathscr{S}^{*}$ with extremal slope. In particular the $\bar{\delta}_{j}$ need not be analytic at the singular points of $\mathscr{S}^{*}$, see Figure 3 .

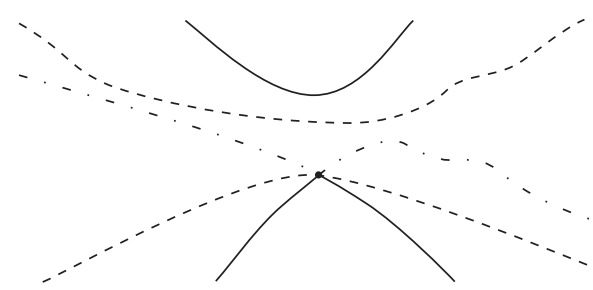

FIgURE 3. The first (continuous), second (dashed) and odd (dot-dashed) tangential loops of the Siebeck curve of Figure 1, sketched in an affine chart of $\mathbf{P}^{\vee}$ according to the conventions set at the beginning of Section 5 .

For each $j$ with $0<j<m / 2$, the arcs $\bar{\delta}_{j}$ and $\bar{\delta}_{m-j}$ have both the same endpoints, namely $q_{j}$ and $q_{m-j}$. They compose thus a closed arc $\sigma_{j}$, with improper points $q_{j}$ and $q_{m-j}$, we name the $j$-th loop of $\mathscr{S}^{*}$ (and also the $j$ th tangential loop of $\mathscr{S}$ ). If $m$ is even, then $\bar{\delta}_{m / 2}$ has coincident endpoints. Therefore it is a further closed arc with only improper point $p_{m / 2}$. We will call it the odd loop of $\mathscr{S}^{*}$ (and also the odd tangential loop of $\mathscr{S}$ ). To unify the notations, we will write $\bar{\delta}_{m / 2}=\sigma_{m / 2}$. The above loops $\sigma_{j}, 1 \leq j<m / 2$, will be referred to as the even loops, to distinguish them from the odd one.

REMARK 5.6. If no three different roots of $f$ are aligned, then, by 5.4, the $\operatorname{arcs} \bar{\delta}_{j}$ and $\bar{\delta}_{m-j}, j<m / 2$, composing each even loop are disjoint. Then the even loops are simple (i.e., with no self-intersections) continuous closed curves, and obviously so is the odd loop in all cases, provided $m$ is even. If there are three aligned roots, the arcs composing an even loop may have common points, which we will call the self-intersecting points of the loop. There is an obvious occurrence of self-intersecting points in the collinear case, as then the line of $\mathrm{P}$ containing all roots belongs, as a point of $\mathrm{P}^{\vee}$, to all the $\bar{\delta}_{j}$.

REMARK 5.7. The loops $\sigma_{j}, 1 \leq j \leq m / 2$, cover the set of all real points of $\mathscr{S}^{*}$. If no three roots are aligned, they are the connected components of $\mathscr{S}^{*}$, by 5.6 .

REMARK 5.8. By 5.4, a simple point $p$ of $\mathscr{S}^{*}$ lies on a unique loop, both $\mathscr{S}^{*}$ and this loop having the same points in a neighbourhood of $p$. If $p$ is a singular point of $\mathscr{S}^{*}$, then it may lie on many loops, each of them being composed in a neighbourhood of $p$ by half-branches of possibly different branches of $\mathscr{S}^{*}$ 
(5.5). Due to this fact, if $\mathscr{S}^{*}$ has singular points, the loops introduced here do not correspond with the connected components of a non-singular model of $\mathscr{S}^{*}$ (the circuits composing $\mathscr{S}^{*}$, in the terminology of [5]). See Figure 3.

In the sequel we will say that a branch $\gamma$ of $\mathscr{S}^{*}$ is partially contained in the loop $\sigma_{j}$ if and only if at least one of the half-branches of $\gamma$ is contained in $\sigma_{j}$.

For each $j, 1 \leq j<m / 2$, take $I_{j}$ to be the set of all improper points whose absolute coordinate is either $\infty$, or strictly greater than $\bar{\beta}_{j}$, or strictly smaller than $\bar{\beta}_{m-j}$. In other words, from the two open segments determined by $q_{j}, q_{m-j}$ in $\Lambda_{\infty}, I_{j}$ is the one containing $\Omega$. Then take

$$
V_{j}=\left\{p=(x, y) \in \mathrm{A} \mid y>\beta_{j}(x)\right\} \cup\left\{p=(x, y) \in \mathrm{A} \mid y<\beta_{m-j}(x)\right\} \cup I_{j} .
$$

It is clear that $\Omega \in V_{j}$ for $1 \leq j<m / 2$, and also that $V_{j-1} \subset V_{j}$ for $2 \leq j<m / 2$.

Lemma 5.9. Any line of $\mathrm{P}^{\vee}$ containing a point of $V_{j}$ contains either two distinct points or a self-intersecting point of $\sigma_{j}$.

Proof. The cases of the improper and the vertical lines being clear, we assume our line $\ell$ to have the equation $y=M x+N$. We have $\bar{\beta}_{j}>\bar{\beta}_{m-j}$; assume first $M>\bar{\beta}_{j}$. Then

$$
\lim _{x \rightarrow+\infty}\left(M x+N-\beta_{j}(x)\right)=\lim _{x \rightarrow+\infty} x\left(M+\frac{N}{x}-\frac{\beta_{j}(x)}{x}\right)=+\infty,
$$

due to the fact that $\lim _{x \rightarrow+\infty}\left(\beta_{j}(x) / x\right)=\bar{\beta}_{j}$ (5.3). Similarly,

$$
\lim _{x \rightarrow-\infty}\left(M x+N-\beta_{j}(x)\right)=-\infty .
$$

This shows that $\ell$ has at least one point $(x, y)$ with $y=\beta_{j}(x)$, and so belonging to $\delta_{j}$. The same equalities hold if $\beta_{m-j}(x)$ is taken instead of $\beta_{j}(x)$, proving that $\ell \cap \delta_{m-j} \neq \emptyset$, and hence the claim in this case.

A similar argument covers the case $M<\bar{\beta}_{m-j}$, so we will assume $\bar{\beta}_{m-j} \leq$ $M \leq \bar{\beta}_{j}$ in the sequel. Then, due to these inequalities, $\ell \cap I_{j}=\emptyset$ and the point of $\ell$ in $V_{j}$ is a proper one, say with coordinates $x_{0}, y_{0}$, and so that either

$$
y_{0}=M x_{0}+N>\beta_{j}\left(x_{0}\right) \quad \text { or } \quad y_{0}=M x_{0}+N<\beta_{m-j}\left(x_{0}\right) .
$$

We will assume that the first inequality holds. The other case may be dealt with similarly, using $\bar{\delta}_{m-j}$ instead of $\bar{\delta}_{j}$. If $M<\bar{\beta}_{j}$, arguing as above

$$
\lim _{x \rightarrow+\infty}\left(M x+N-\beta_{j}(x)\right)=\lim _{x \rightarrow+\infty} x\left(M+\frac{N}{x}-\frac{\beta_{j}(x)}{x}\right)=-\infty
$$


while for $\bar{\beta}_{m-j}<M$,

$$
\lim _{x \rightarrow-\infty}\left(M x+N-\beta_{j}(x)\right)=\lim _{x \rightarrow-\infty} x\left(M+\frac{N}{x}-\frac{\beta_{j}(x)}{x}\right)=-\infty .
$$

If $\bar{\beta}_{m-j}<M<\bar{\beta}_{j}$, then both (6) and (7) hold and, together with the first inequality in (5), they assure that there are two different points of $\bar{\delta}_{j}$ on $\ell$. In case of being $\bar{\beta}_{m-j}=M$ or $M=\bar{\beta}_{m}$, then the improper point of $\ell$ belongs to $\bar{\delta}_{j}$ and the existence of a second intersection point results as above from either (6) or (7), one of them being still true because $\bar{\beta}_{j} \neq \bar{\beta}_{m-j}$.

LeMma 5.10. If $m$ is even, then any line of $\mathrm{P}^{\vee}$ contains at least one point of the odd loop $\sigma_{m / 2}$.

Proof. Left to the reader, who may proceed like in 5.9.

Proposition 5.11. Any real line of $\mathrm{P}^{\vee}$ containing a point of $V_{1}$ has a total of $m-1$ real intersection points with $\mathscr{S}^{*}$, each counted as many times as the number of real tangents to $\mathscr{S}^{*}$ at the intersection point.

Proof. Let $\ell$ be a line through a point in $V_{1}$ : since this point does not belong to $\mathscr{S}^{*}, \ell \not \subset \mathscr{S}^{*}$. By Lemmas 5.9 and 5.10, adding up the numbers of intersection points of $\ell$ and each of the loops composing $\mathscr{S}^{*}$, self-intersecting points counted twice, gives a total of at least $m-1$ intersection points. Let us check how many times each point $p \in \ell \cap \mathscr{S}^{*}$ appears repeated in this count. Let $\tau_{p}$ be the multiplicity of $\mathscr{S}^{*}$ at $p\left(\tau_{p}=e_{p}-1\right.$ if $p$ is singular); since the real singularities of $\mathscr{S}^{*}$ are ordinary (2.4), $\tau_{p}$ is also the number of tangents to $\mathscr{S}^{*}$ at $p$. By $5.4, p$ belongs to exactly $\tau_{p}$ of the arcs $\bar{\delta}_{j}$, that is to $\tau_{p}$ loops if we agree in counting twice the loops for which $p$ is a self-intersecting point. Thus, it turns out that

$$
m-1 \leq \sum_{p} \tau_{p} \leq \sum_{p}\left[\ell \cdot \mathscr{S}^{*}\right]_{p} \leq m-1,
$$

where the summations are over the real points $p \in \ell \cap \mathscr{S}^{*}$ and the last inequality is due to Bezout's theorem. Hence

$$
m-1=\sum_{p} \tau_{p}
$$

as wanted.

Corollary 5.12. No real line of $\mathrm{P}^{\vee}$ containing a point of $V_{1}$ has an imaginary intersection with $\mathscr{S}^{*}$, or is tangent to it. 
Proof. For each $p \in \mathscr{S}^{*}$, still denote by $\tau_{p}$ the multiplicity of $\mathscr{S}^{*}$ at $p$; as recalled above, $\tau_{p}$ is the number of tangents to $\mathscr{S}^{*}$ at $p$. If $\ell$ is a line containing a point of $V_{1}$, it is not contained in $\mathscr{S}^{*}$ and Bezout's theorem gives

$$
m-1 \geq \sum_{p \in \ell \cap \mathscr{S}^{*}}\left[\ell \cdot \mathscr{S}^{*}\right]_{q} \geq \sum_{p \in \ell \cap \mathscr{S}^{*}} \tau_{p}=m-1
$$

where the summations are over real points and the last equality is due to 5.11. The two inequalities above are thus equalities: the first one guarantees the absence of imaginary intersections, while the second one assures that there are no contact points.

REMARK 5.13. The count made in the proof of 5.11 shows that, by counting twice the self-intersecting points, a line containing a point of $V_{1}$ contains exactly two points of each even loop, and one point of the odd loop if $m$ is even.

We will say that a line $t$ of $\mathrm{P}^{\vee}$ is tangent to the loop $\sigma_{j}$ of $\mathscr{S}^{*}$ at a point $p \in \sigma_{j}$, if and only if $t$ is tangent to a branch of $\mathscr{S}^{*}$ at $p$ partially contained in $\sigma_{j}$. We are interested in the tangents to the first loop $\sigma_{1}$ only. For them, the facts quoted below for further reference are quite clear:

REMARK 5.14. If $p \in \sigma_{1}$ is a singular point of $\mathscr{S}^{*}$, then there are just two tangents to $\sigma_{1}$ at $p$ : these are the tangents to $\mathscr{S}^{*}$ at $p$ with extremal slopes. If, otherwise, $p$ is a non-singular point of $\mathscr{S}^{*}$, then the tangent to $\mathscr{S}^{*}$ at $p$ is the only tangent to $\sigma_{1}$ at $p$.

Still regarding the first loop, we have:

LEMMA 5.15. If not all roots of $f$ are collinear and $\mathscr{S}$ has $p$ as a nested point, then $p^{*}$ and the first loop of $\mathscr{S}^{*}$ have empty intersection.

Proof. Since the collinear case has been excluded, we may pick two different lines of $\mathrm{P}, \ell_{1}, \ell_{2}$, among those which, according to 4.1, go through $p$ and contain the roots of $f$. Then $\ell_{1} \in p^{*}$ and there are two or more lines $z_{j}^{*}$ going through $\ell_{1}$. From them let $z_{j_{1}}^{*}$ and $z_{j_{2}}^{*}$ be those with minimal and maximal slope. If the slopes of $p^{*}, z_{j_{1}}^{*}$ and $z_{j_{2}}^{*}$ are $\varepsilon, \varepsilon_{1}, \varepsilon_{2}$, respectively, then, by condition (2) of 4.1, $\varepsilon_{1}<\varepsilon<\varepsilon_{2}$. Similarly, there are $z_{j_{3}}^{*}$ and $z_{j_{4}}^{*}$ going through $\ell_{2}$, with extremal slopes $\varepsilon_{3}$ and $\varepsilon_{4}$, so that $\varepsilon_{3}<\varepsilon<\varepsilon_{4}$. Fix any $x \in \mathrm{R}$ and let $q_{x}=(x, h(x))$ be the point of $p^{*}$ with abscissa $x$. On two of the lines selected above the points with abscissa $x$ have their ordinates greater than $h(x)$, and at least one of these ordinates is strictly greater than $h(x)$. Then, by 5.1 , there is $j$ for which $\beta_{j}(x)>h(x)$ and so $\beta_{1}(x)>h(x)$. This proves that the points on $p^{*}$ and $\bar{\delta}_{1}$ with abscissa $x$ are different. The same happens with the points 
on $\Lambda_{\infty}$, as by 5.1,(b) there is one $\bar{\beta}_{j}$ between $\varepsilon_{1}$ and $\varepsilon_{3}$ which assures $\bar{\beta}_{1}>\varepsilon$. As a result $\bar{\delta}_{1} \cap p_{*}=\emptyset$. A similar argument, this time using points below $p^{*}$, shows that $\bar{\delta}_{m-1} \cap p^{*}=\emptyset$ and hence completes the proof.

Next comes a similar result to 5.11, this time relative to the tangents to the first loop; we will make use of it later on.

Proposition 5.16. Assume not to be in the collinear case and that $p$ is a point of $\sigma_{1}$ which has multiplicity $\tau_{p}$ on $\mathscr{S}^{*}$. Let $t$ be a line tangent to $\sigma_{1}$ at $p$. Then the number of real intersection points of $t$ and $\mathscr{S}^{*}$ other than $p$ is $m-\tau_{p}-2$, each intersection point $q$ being counted as many times as the number of real tangents to $\mathscr{S}^{*}$ at $q$.

Proof. Note first that $t$ cannot be contained in $\mathscr{S}^{*}$, as in such a case it would be $t=q^{*}, q$ a nested point, and $p \in t \cap \sigma_{1}$, against 5.15. Exclude for a while the cases $m=3,4$. Then $V_{2}$ is defined and we claim that $t$ contains points of it. Indeed, this is clear if $p$ is a non-singular point of $\mathscr{S}^{*}$, as then $p \in V_{2}$. Otherwise the slope of $t$ is extremal among the slopes of the tangents to $\mathscr{S}^{*}$ at $p$ (5.14), which assures that all points in one of the half-lines composing $t-p$ and close enough to $p$ belong to $V_{2}$.

According to 5.9 and 5.10, we have at least two real intersection points of $t$ with each of the even loops from the second one onwards, self-intersecting points being counted twice, and at least a further one with the odd loop in case it exists. This makes a total of at least (maybe repeated) $m-3$ intersection points. Since this is clearly also true for $m=3,4$, we remove the above restriction $m>4$ from now on. From the intersection points of $t$ and the loops other than $\sigma_{1}, \tau_{p}-1$ are equal to $p$, as there are $\tau_{p}-1 \operatorname{arcs} \bar{\delta}_{j}, 1<j<m-1$ containing $p$. As in the proof of 5.11, each $q \neq p, q \in t \cap \mathscr{S}^{*}$, appears at least $\tau_{q}$ times among these intersection points, $\tau_{q}$ being the multiplicity, and also the number of tangents, of $\mathscr{S}^{*}$ at $q$. Then,

$$
m-\tau_{p}-2=m-3-\left(\tau_{p}-1\right) \leq \sum_{\substack{q \in t \cap \mathscr{S}^{*} \\ q \neq p}} \tau_{q} \leq \sum_{\substack{q \in t \cap \mathscr{S}^{*} \\ q \neq p}}\left[t \cdot \mathscr{S}^{*}\right]_{q} \leq m-\tau_{p}-2,
$$

where the last inequality follows from Bezout's theorem and the fact that $t$ is tangent to $\mathscr{S}^{*}$ at $p$ and therefore $\left[t \cdot \mathscr{S}^{*}\right]_{p} \geq \tau_{p}+1$.

COROLlary 5.17. All branches of $\mathscr{S}^{*}$ partially contained in the first loop have class one and share its tangent with no other branch of $\mathscr{S}^{*}$.

Proof. Assume that the branch has origin $p$ and tangent $t$. Then $\left[t \cdot \mathscr{S}^{*}\right]_{p} \geq$ 
$\tau_{p}+1$. On the other hand, from Bezout's theorem and 5.16,

$$
\begin{aligned}
{\left[t \cdot \mathscr{S}^{*}\right]_{p} } & \leq m-1-\sum_{\substack{q \in t \cap \mathscr{S}^{*} \\
q \neq p}}\left[t \cdot \mathscr{S}^{*}\right]_{q} \leq m-1-\sum_{\substack{q \in t \cap \mathscr{S}^{*} \\
q \neq p}} \tau_{q} \\
& =m-1-\left(m-\tau_{p}-2\right)=\tau_{p}+1 .
\end{aligned}
$$

It follows then that $\left[t \cdot \mathscr{S}^{*}\right]_{p}=\tau_{p}+1$ and the first claim is proved. This equality also proves that no other branch of $\mathscr{S}^{*}$ with origin at $p$ has tangent $t$, which is also clear from being $p$ either a simple or an ordinary multiple point of $\mathscr{S}^{*}$. The same equality and 5.16 give

$$
\left[t \cdot \mathscr{S}^{*}\right]_{p}+\sum_{\substack{q \in t \cap \mathscr{S}^{*} \\ q \neq p}} \tau_{q}=m-1 .
$$

Using Bezout's theorem once again, this assures that $\left[t \cdot \mathscr{S}^{*}\right]_{q}=\tau_{q}$ for any $q \in \cap \mathscr{S}^{*}, q \neq p$, and so $t$ is not tangent to $\mathscr{S}^{*}$ at any of these $q$.

\section{Lines tangent to $\mathscr{S}$}

The pencils of parallel lines of $P$ are the lines of $P^{\vee}$ through $\Omega$. Using the affine chart $A$ and the conventions set in section 5, the pencils of parallel lines of $E$ are the improper line and the vertical lines of $A$. The improper point of each vertical line being $\Omega$, we see that the affine structure that $A$ induces on a vertical line is just its natural affine structure as a pencil of parallel lines. In particular, the betweenness relation on points of a vertical line agrees with their betweenness as parallel lines. The second affine coordinate of the points of each vertical line may be taken as an affine coordinate on the line seen as a pencil of parallel lines of $E$. Also the absolute coordinate taken on $\Lambda_{\infty}$ is an affine coordinate in $\Lambda_{\infty}$ seen as a pencil of parallel lines.

We call a tangent $\ell$ to an augmented curve $\mathscr{C}$ extremal if and only if $\ell$ is a proper line and does not lie between other two proper lines tangent to $\mathscr{C}$ and parallel to $\ell$.

By the above and the definition of the loops, we see that the extremal tangents to $\mathscr{S}$ in the direction of an improper point $p$ are the intersections of $p^{*}$ and the first tangential loop of $\mathscr{S}$. The number of distinct extremal tangents in a given direction is exactly two, but for a special situation arising in the collinear case only, namely:

LemMa 6.1. There are exactly two distinct extremal tangents to $\mathscr{S}$ in a given direction $w$, but for the case in which a line in the direction $w$ contains all roots of $f$. Then such a line is the only extremal tangent in the direction $w$. 
Proof. By 5.14, there are two distinct extremal tangents for each direction $w$, but for the case in which a line $\ell$ in the direction $w$ is a self-intersecting point of the first loop. If this is the case, by $5.1, \ell$ belongs to all loops and is a self-intersecting point of the even ones; by 5.4, this assures that $\ell$ contains all roots of $f$, after which the rest of the claim is clear.

In the sequel, the only extremal tangent in the exceptional case of 6.1 will be taken as a pair of coincident extremal tangents.

According to its definition in Section 5, the open set $V_{1} \subset \mathrm{P}^{\vee}$ is composed of the improper line and the proper lines lying outside the extremal tangents in their directions. The lines $\ell \in V_{1}$ will be called outer lines in the sequel. We will see in 6.3 below that they do not intersect $\mathscr{S}$ in real points. This is false in general, even for bounded curves, the astroid providing an easy example.

Using these terms, by duality, 5.11, 5.12 and 5.15 translate into:

PROPOSITION 6.2. The number of real tangents to $\mathscr{S}$ from any point of any outer line is $m-1$, each tangent counted as many times as the number of its different real contact points.

Corollary 6.3. No real point of an outer line belongs to $\mathscr{S}$ or has an imaginary tangent to $\mathscr{S}$ through.

Proposition 6.4. If not all roots of $f$ are aligned and $\mathscr{S}$ has $p$ as nested point, then no extremal tangent to $\mathscr{S}$ goes through $p$.

We close this section presenting a further property of the Siebeck curve. As in former cases, it directly follows from the results of Section 5.

Proposition 6.5. Both the contact points of the simple extremal tangents to $\mathscr{S}$ and the extremal contact points of the multiple extremal tangents to $\mathscr{S}$ are non-singular points of the one-dimensional part of $\mathscr{S}$.

Proof. By 6.4, a contact point of an extremal tangent is not a nested point, and so it belongs to the one-dimensional part of $\mathscr{S}$. Assume that $q$ is an extremal contact point of an extremal tangent $t$ to $\mathscr{S}$ (the only contact point if $t$ is a simple tangent). Then $t$ belongs to the first tangential loop $\sigma_{1}$ of $S$ and $q^{*}$ is a tangent to $\mathscr{S}^{*}$ at $t$ with extremal slope. Hence, by $5.14, q^{*}$ is a tangent to $\sigma_{1}$. By $5.17, q^{*}$ has no other contact point with $S^{*}$ and the only branch of $\mathscr{S}^{*}$ tangent to $q^{*}$ has class one. By duality, this assures that there is a single branch of the one-dimensional part of $\mathscr{S}$ with origin at $q$ and that the multiplicity of this branch is one, hence the claim. 


\section{First location of roots}

We are now able to prove Linfield's location of roots. The situation in the collinear case being clear (see Example 2.6), we exclude it for simplicity.

THEOREM 7.1 (Linfield). The complex field $\mathrm{C}$ being viewed as an Euclidean plane, assume that $f \in \mathrm{C}[z]$ has different roots $z_{1}, \ldots, z_{m}, m \geq 3$, noncollinear and with respective multiplicities $\mu_{1}, \ldots, \mu_{m}$. Fix an arbitrary direction $w$ on $C$ and consider the group $L=\mu_{1} \ell_{w, 1}+\cdots+\mu_{m} \ell_{w, m}$ of the lines $\ell_{w, j}$ projecting the roots $z_{j}$ in the direction $w$, each with multiplicity equal to the sum of the multiplicities of the roots lying on it. Let $\ell_{w}, \ell_{w}^{\prime}$ be the extremal lines among those in the direction $w$ which either belong to the harmonic group of $L$ or contain two or more roots of $f$. Then all roots of $d f / d z$ which are not roots of $f$ lie in the open stripe bounded by $\ell_{w}$ and $\ell_{w}^{\prime}$.

Proof. By 6.3 and 3.1, the points from which there are imaginary tangents to the one-dimensional part $\tilde{\mathscr{S}}$ of $\mathscr{S}$ belong to the open stripe $\Sigma_{w}$ bounded by the extremal tangents to $\mathscr{S}$ in the direction $w$. Thus, by 4.7, a nested point of $\mathscr{S}$ belongs to $\Sigma_{w}$. The same is true for the foci of $\tilde{\mathscr{S}}$ as, by its definition, the lines joining them to the cyclic points are tangent to $\tilde{\mathscr{S}}$. All foci of $\mathscr{S}$ belong thus to $\Sigma_{w}$.

Now, on one hand, the roots of $d f / d z$ other than the roots of $f$ are the foci of $\mathscr{S}$ by 2.1. On the other, by 2.2, the extremal tangents to $\mathscr{S}$ in the direction $w$ are the lines $\ell_{w}, \ell_{w}^{\prime}$ in the claim. Putting these facts all together completes the proof.

REMARK 7.2. If not all roots are aligned, notations are as in 7.1, still $\Sigma_{w}$ is the open stripe bounded by $\ell_{w}, \ell_{w}^{\prime}$ and we take $\Sigma=\bigcap_{w} \Sigma_{w}$, then 7.1 asserts that all roots of $d f / d z$ other than the roots of $f$ belong to $\Sigma$.

Remark 7.3. With the notations being as in 7.1, if the lines $\ell_{w, j}$ have equations $a x+b y+c_{j}=0$ and we take $g(X)=\prod_{j}\left(X-c_{j}\right)^{\mu_{j}}$, then the lines $\ell_{w}, \ell_{w}^{\prime}$ are the extremal ones among those with equations $a x+b y+c=0$, for $c$ a root of $d g / d X$.

A couple of facts seen while proving 7.1 are worth separate claims, namely:

COROLlaRy 7.4 (of the proof of 7.1). If not all roots of $f$ are aligned and the notations are as in 7.1, then $\ell_{w}, \ell_{w}^{\prime}$ are the extremal tangents to $\mathscr{S}$ in the direction $w$.

COROLlaRY 7.5 (of the proof of 7.1). If not all roots of $f$ are aligned, then the foci of $\mathscr{S}$ lie in the open stripe bounded by any pair of extremal tangents to $\mathscr{S}$.

Next we compare Linfield's and Gauss-Lucas' locations. We need: 
Lemma 7.6. Assume that a line $\ell$ of $\mathrm{E}$ joins two different roots of $f$. Then $\ell$ is an extremal tangent to $\mathscr{S}$ if and only if one of the open half-planes determined by $\ell$ contains no root of $f$ or, equivalently, if and only if $\ell$ contains a side of the boundary of the convex hull of the roots of $f$.

Proof. The line $\ell$ is tangent to $\mathscr{S}$ by 2.1 . By 2.2 , the tangents to $\mathscr{S}$ parallel to $\ell$ either are lines joining two roots or lie between two lines projecting roots in the direction of $\ell$, and there is one of the latter for each pair of consecutive distinct lines projecting roots, hence the claim.

PROPOSITION 7.7. If not all roots of $f$ are aligned, then the set $\Sigma$ of 7.2 is strictly contained in the interior of the convex hull of the roots of $f$.

Proof. Let $s_{1}, \ldots, s_{r}$ be the lines containing one of the sides of the convex hull of the roots. For each $j, s_{j}$ is an extremal tangent to $\mathscr{S}$ by 7.6 and hence (7.4) one of the lines $\ell_{w}, \ell_{w}^{\prime}$ of 7.1, say $s_{j}=\ell_{w}$. From the two open half-planes determined by $s_{j}$, let $H_{j}$ be the one containing the roots of $f$ lying not on $s_{j}$. By the definition of $\ell_{w}, \ell_{w}^{\prime}, H_{j}$ contains $\ell_{w}^{\prime}$ and therefore also the open stripe $\Sigma_{w}$. Since $H_{1} \cap \cdots \cap H_{r}$ is the interior of the convex hull of the roots, the claimed inclusion follows.

Assume that $w$ a direction other than those of the lines joining two roots of $f$. Assume also that the extremal lines projecting the roots of $f$ in the direction $w$ are going through the roots $z_{j}$ and $z_{j^{\prime}}$. Then, by the separation property of the harmonic group, $z_{j}$ and $z_{j^{\prime}}$ lie outside the closed stripe bounded by $\ell_{w}, \ell_{w}^{\prime}$, which shows that the above inclusion is strict.

We close this section by presenting a characterization of the set $\Sigma$ in terms of the Siebeck curve:

Proposition 7.8. The set $\Sigma$, defined in 7.2, equals the interior of the convex hull of the Siebeck curve $\mathscr{S}$.

Proof. For this proof, we place ourselves in the Euclidean plane $E$ of the complex numbers. The reader is referred to any book on convex sets, for instance [11], for the definitions and results regarding separation used below. Let $\mathrm{CH}(\mathscr{S})$ be the convex hull of $\mathscr{S}$. We already know $([4], 8.6)$ that $\mathscr{S}$ has no improper points and therefore is compact, after which $\mathrm{CH}(\mathscr{S})$ is closed. If $s$ is an extremal tangent to $\mathscr{S}$, then let $\bar{H}_{s}$ be the closed half-plane with boundary $s$ containing all the points of $\mathscr{S}(6.3)$. Since $\mathscr{S}$ is convex, $\bar{H}_{s}$ contains also $\mathrm{CH}(\mathscr{S})$. Furthermore the boundary $s$ of $\bar{H}_{s}$ contains a point of $\mathrm{CH}(\mathscr{S})$, namely any of the contact points of $s$. This proves that $\bar{H}_{s}$ is a supporting hyperplane of $\mathrm{CH}(\mathscr{S})$. If $s$ is not an extremal tangent, then it lies either between or outside the extremal tangents parallel to it. In the first case there are points of $\mathrm{CH}(\mathscr{S})$ (the contact points of the extremal tangents) on both sides of $s$. In the second case, $s$ 
contains no point of $\mathrm{CH}(\mathscr{S})$ as, by $6.3, \mathrm{CH}(\mathscr{S})$ is contained in the closed stripe bounded by the extremal tangents parallel to $s$. As a consequence, none of the half-planes determined by $s$ supports $\mathrm{CH}(\mathscr{S})$. The supporting half-planes of $\mathrm{CH}(\mathscr{S})$ are thus just the $\bar{H}_{s}$ for $s$ an extremal tangent to $S$. Then, as for any closed convex set, $\mathrm{CH}(\mathscr{S})$ is the intersection of its supporting half-planes ([11], 1.3.5), that is,

$$
\mathrm{CH}(\mathscr{S})=\bigcap_{s \in \sigma_{1}} \bar{H}_{s}
$$

If we take $H_{s}=\bar{H}_{s}-s$, then, on one hand, directly from its definition,

$$
\Sigma=\bigcap_{s \in \sigma_{1}} H_{s}
$$

On the other, an easy argument using equality (8) and the fact that any point in the boundary of a closed convex set belongs to the boundary of one of its supporting half-planes ([11], 1.3.2), allows to prove that the interior of $\mathrm{CH}(\mathscr{S})$ is $\bigcap_{s \in \sigma_{1}} H_{s}$ which, together with (9), proves the claim.

\section{Second location of roots}

From now on we assume not to be in the collinear case. As recalled above, the Siebeck curve $\mathscr{S}$ has no improper (real) points ([4], 8.6). Among the connected components of $\mathrm{P}-\mathscr{S}$ there is thus a well determined one containing the improper line $\Omega$ : we call it the exterior of $\mathscr{S}$, denoted $\mathbf{E}(\mathscr{S})$ in the sequel. If $\overline{\mathbf{E}(\mathscr{S})}$ is the closure of $\mathbf{E}(\mathscr{S})$, the second location of the roots of the derivative is:

THEOREM 8.1. No root of $d f / d z$ other than the roots of $f$ belongs to $\overline{\mathbf{E}(\mathscr{S})}$.

Proof. Let $m^{\prime}$ be the class of the one-dimensional part $\tilde{\mathscr{S}}$ of $\mathscr{S}$ : either $m^{\prime}=m-2$, if $\mathscr{S}$ has a nested point, or, otherwise, $m^{\prime}=m-1$. Let $p$ be a point in $\mathbf{E}(\mathscr{S})$; it may be joined to a point $q$ on the improper line by a piecewise linear path $\Gamma$ made by finitely many closed segments of line disjoint with $\mathscr{S}$. In particular exception (b) of 3.4(3) does not apply to any point of $\Gamma$. Neither does exception (a) of 3.4(3), as $\mathscr{S}^{*}$ has no real singularities other than ordinary ones (2.4) and so all real branches of $\tilde{\mathscr{S}}$ have class one. Thus, each segment composing $\Gamma$ may be covered by finitely-many open intervals, each satisfying the claims of 3.4 relative to $\tilde{\mathscr{S}}$. On the other hand, by 6.2 , the maximal number $m^{\prime}$ of real tangents from a point to $\tilde{\mathscr{S}}$ is reached at all points in an open neighbourhood of $q$. Then repeated applications of 3.4, always with $v_{-}=v_{0}=v_{+}$due to the maximality of $m^{\prime}$, prove that there are $m^{\prime}$ real tangents 
from $p$ to $\tilde{\mathscr{S}}$, and so no imaginary one. The same is true for any $p \in \overline{\mathbf{E}(\mathscr{S})}$, by 3.1. Thus, arguing as the proof of 7.1, no $p \in \overline{\mathbf{E}(\mathscr{S})}$ may be a root of $d f / d x$ other than the roots of $f$.

For future reference let us retain:

COROLlary 8.2 (of the proof of 8.1). There are no imaginary tangents to the one-dimensional part of $\mathscr{S}$ from any point in $\overline{\mathbf{E}(\mathscr{S})}$.

The present location is not worse than the one of Section 7:

Proposition 8.3. It holds $\Sigma \supset \mathrm{P}-\overline{\mathbf{E}(\mathscr{S})}$.

Proof. For each direction $w$, the set of all points on outer lines in the direction $w$ is connected, contains $\Omega$ and, by 6.3 , is disjoint with $\mathscr{S}$. It is thus contained in $\mathbf{E}(\mathscr{S})$ and therefore its closure $\mathrm{P}-\Sigma_{w}$ is contained in $\overline{\mathbf{E}(\mathscr{S})}$. By the arbitrariness of $w, \mathrm{P}-\Sigma \subset \overline{\mathbf{E}(\mathscr{S})}$, hence the claim.

We will pay some attention to the case in which no side of the convex hull of the roots of $f$ contains three different roots, as it will turn out to be the case in which the inclusion of 8.3 is an equality, and therefore the location of 8.1 is equivalent to Linfield's one. The outer part of the Siebeck curve has, in this case, nice regularity properties, namely:

PROPOSITION 8.4. If no side of the convex hull of the roots of $f$ contains three different roots, then the contact points of the extremal tangents to the Siebeck curve of $f$ are the points of an analytic, smooth, closed and convex curve of $\mathrm{E}$ which is a connected component of $\mathscr{S}$ and whose interior (as a Jordan curve) contains all the other connected components of $\mathscr{S}$.

Proof. By 2.4 the only singularities of $\mathscr{S}^{*}$ are the lines containing three or more roots, and from these, by 7.6, the extremal tangents are those containing a side of the convex hull of the roots. The hypothesis is thus satisfied if and only if no extremal tangent is a singular point of $\mathscr{S}^{*}$ (that is, a multiple tangent). The extremal tangents being the points of the first loop $\sigma_{1}$ of $\mathscr{S}^{*}, \sigma_{1}$ contains no singular point of $\mathscr{S}^{*}$ and in particular shares no point with any of the other loops. Therefore $\sigma_{1}$ is a connected component of $\mathscr{S}^{*}$ and also a smooth and closed analytic curve of $\mathrm{P}^{\vee}$.

Fix homogeneous coordinates $x_{0}, x_{1}, x_{2}$ on $\mathrm{P}$, their dual ones $u_{0}, u_{1}, u_{2}$ on $\mathrm{P}^{\vee}$ and an equation $F=0, F \in \mathrm{R}\left[u_{0}, u_{1}, u_{2}\right]$, of $\mathscr{S}^{*}$. It is well known that the relations

$$
x_{0}=\frac{\partial F}{\partial u_{0}}, \quad x_{1}=\frac{\partial F}{\partial u_{1}}, \quad x_{2}=\frac{\partial F}{\partial u_{2}}
$$

define a rational map

$$
\Psi: \mathrm{P}^{\vee} \longrightarrow \mathrm{P}
$$


which maps each non-singular point of $\mathscr{S}^{*}$ to its contact point (as a simple tangent to $\mathscr{S}$ ) and remains undefined at the singular points of $\mathscr{S}^{*}$ (the dual assertion is more usual, see [13], V.8.1 or [6], 5.3 ). Since $\sigma_{1}$ contains no singular point of $\mathscr{S}^{*}, \Psi$ restricts to an analytic map defined in an open set containing $\sigma_{1}$ and $\mathscr{S}_{1}=\Psi\left(\sigma_{1}\right)$ is the set of contact points of the extremal tangents to $\mathscr{S}$. Furthermore, have seen in 5.17 that no point of $\sigma_{1}$ is a flex of $\mathscr{S}^{*}$, therefore the Hessian determinant

$$
\left|\frac{\partial^{2} F}{\partial u_{i} \partial u_{j}}\right|
$$

does not vanish at any point of $\sigma_{1}$. A straightforward computation relates the above Hessian to the Jacobian of $\Psi$ at each $p \in \sigma_{1}$ and proves the restriction of $\Psi$ to be a local isomorphism at each point of $\sigma_{1} . \mathscr{S}_{1}=\Psi\left(\sigma_{1}\right)$ is thus a smooth, closed and connected analytic curve of E contained in $\mathscr{S}$. Since $\mathscr{S}$ has no flexes (2.5), $\mathscr{S}_{1}$ is in addition convex.

We know that all points of $\mathscr{S}_{1}$ are non-singular for $\mathscr{S}$ (6.5), after which there is a single branch of $\mathscr{S}$ at each $q \in \mathscr{S}_{1}$. By the curve selection lemma ([2], 8.1.13, for instance) no $q \in \mathscr{S}_{1}$ may then be adherent to $\mathscr{S}-\mathscr{S}_{1}$, and $\mathscr{S}_{1}$ is a connected component of $\mathscr{S}$.

Jordan's curve theorem asserts that $\mathrm{P}-\mathscr{S}_{1}$ has two connected components, both with boundary $\mathscr{S}_{1}$. From them, one contains the improper line and is called the exterior of $\mathscr{S}_{1}$ and the other is called the interior of $\mathscr{S}_{1}$; they will be denoted $\operatorname{Ext}\left(\mathscr{S}_{1}\right)$ and $\operatorname{Int}\left(\mathscr{S}_{1}\right)$, respectively. The set $\mathscr{S}_{1} \cup \operatorname{Int}\left(\mathscr{S}_{1}\right)$ is then closed and convex, and its supporting half-planes are those with boundary a tangent to $\mathscr{S}_{1}$ and containing $\mathscr{S}_{1}$ ([1], 9.6.2, for instance). Since the tangents to $\mathscr{S}_{1}$ are the extremal tangents to $\mathscr{S}$, arguing as in the proof of 7.8, $\Sigma=\operatorname{Int}\left(\mathscr{S}_{1}\right)$ and so, by 6.3, all points of $\mathscr{S}-\mathscr{S}_{1}$ lie in $\operatorname{Int}\left(\mathscr{S}_{1}\right)$, which completes the proof.

If three roots lie on the same side of the boundary of the convex hull of the roots of $f$, then, as shown by the example of figure 1 , the structure of the set of contact points of the extremal tangents is more complicated: besides being non-connected and having isolated points, its points do not need to belong all to the same circuit of $\mathscr{S}$.

COROLLARY 8.5 (of the proof of 8.4). If no side of the boundary of the convex hull of the roots of $f$ contains three aligned roots, then $\Sigma=\mathrm{P}-\overline{\mathbf{E}(\mathscr{S})}$.

Proof. In the proof of 8.4 we have seen that $\Sigma=\operatorname{Int}\left(\mathscr{S}_{1}\right)$. Using 8.4, it is direct to check, from their definitions above, that $\mathbf{E}(S)=\operatorname{Ext}\left(\mathscr{I}_{1}\right)$, hence the claim.

Before proving the converse of 8.5 we need: 
LEMMA 8.6. Any simple point of the one-dimensional part of $\mathscr{S}$ is adherent to $\mathrm{P}-\overline{\mathbf{E}(\mathscr{S})}$.

Proof. Assume that $\gamma$ is a branch of a real plane algebraic curve with origin at a point $O$ and both multiplicity and class equal to one. Then, in suitable affine coordinates with origin $O$, it may be represented by a Puiseux parameterization

$$
x=t, \quad y=a t^{2}+\cdots
$$

with $a$ real and non-zero. It is then clear that there are lines parallel and arbitrarily close to the tangent to $\gamma, y=0$, whose intersection with $\gamma$ contains a pair of imaginary points. Our hypothesis on $\gamma$ being selfdual, the dual of the above assures that there exist a certain line $\ell$ through $O$ and points $q \in \ell$, arbitrarily close to $O$, so that there are imaginary tangents to $\gamma$ from each point $q$.

Since $\mathscr{S}$ has no flexes, the above applies to the only branch of the onedimensional part $\tilde{\mathscr{S}}$ of $\mathscr{S}$ at any of its non-singular points $p$, assuring that there are points arbitrarily close to $p$ from which there are imaginary tangents to $\tilde{\mathscr{S}}$; since these points belong to $\mathrm{P}-\overline{\mathbf{E}(\mathscr{S})}$ due to 8.2 , the claim is proved.

Now, for the converse:

PROPOSITION 8.7. If three roots of $f$ lie on the same side of the boundary of the convex hull of all roots of $f$, then the set $\mathrm{P}-\overline{\mathbf{E}(\mathscr{S})}$ is not convex and so, in particular, $\Sigma \neq \mathrm{P}-\overline{\mathbf{E}(\mathscr{S})}$.

Proof. The hypothesis assures the existence of a multiple and extremal tangent $\ell$ to $\mathscr{S}$. Let $p_{1}, p_{2}$ be its extremal contact points, obviously $p_{1} \neq p_{2}$. By its definition, $\mathscr{S}$ does not contain lines and therefore there are finitely many points $p_{1}, \ldots, p_{r}$ of $\mathscr{S}$ on $\ell$. Pick a point $q$ in the segment with endpoints $p_{1}, p_{2}$ and not in $\mathscr{S}$. Since the union of all the outer lines parallel to $\ell$ and $\ell-\left\{p_{1}, \ldots, p_{r}\right\}$ is a connected set, disjoint with $\mathscr{S}$ and containing both $q$ and $\Omega, q \in \mathbf{E}(S)$ and so there is an open neighbourhood $U$ of $q$ contained in $\mathbf{E}(S)$. By 8.6 we may then choose points $p_{1}^{\prime}, p_{2}^{\prime} \in \mathrm{P}-\overline{\mathbf{E}(\mathscr{S})}$ close enough to $p_{1}$ and $p_{2}$, respectively, in order to have the intersection of $U$ and the segment with endpoints $p_{1}^{\prime}, p_{2}^{\prime}$ non empty. This shows that $\mathrm{P}-\overline{\mathbf{E}(\mathscr{S})}$ is not convex.

\section{REFERENCES}

1. Berger, M., and Gostiaux, B., Differential Geometry: Manifolds, Curves and Surfaces, Grad. Texts in Math. 115, Springer, New York 1988.

2. Bochnak, J., Coste, M., and Roy, M. F., Real Algebraic Geometry, Ergebn. Math. Grenzgeb. (3) 36, Springer, Berlin 1998. 
3. Casas-Alvero, E., Singularities of Plane Curves, London Math. Soc. Lecture Note 276, Cambridge Univ. Press, Cambridge 2000.

4. Casas-Alvero, E., Roots of complex polynomials and foci of real algebraic curves, preprint, 2010.

5. Coolidge, J. L., A Treatise on Plane Algebraic Curves, Oxford Univ. Press, London 1931.

6. Fischer, G., Plane Algebraic Curves, Student Math. Library 15, Amer. Math. Soc., Providence, RI 2001.

7. Linfield, B. Z., On certain polar curves with their application to the location of the roots of the derivatives of a rational function, Trans. Amer. Math. Soc, 27 (1920), 17-21.

8. Marden, M., Geometry of Polynomials, 2nd ed., Math. Surveys 3, Amer. Math. Soc., Providence, RI 1966.

9. Milnor, J., Singular Points of Complex Hypersurfaces, Ann. of Math. Studies 61, Princeton Univ. Press, Princeton 1968.

10. Prasolov, V. V., Polynomials, Algorithms and Computation in Math. 11, Springer, Berlin 2004.

11. Schneider, R., Convex Bodies: the Brunn-Minkowski Theory, Encycl. Math. Appl. 44, Cambridge Univ. Press, Cambridge 1993.

12. Siebeck, J., Über eine neue analytische Behandlungweise der Brennpunkte, J. Reine Angew. Math. 64 (1864), 175-182.

13. Walker, R., Algebraic Curves, Dover, New York 1962.

DEPARTAMENT D'ÀLGEBRA I GEOMETRIA

UNIVERSITAT DE BARCELONA

GRAN VIA 585

08007 BARCELONA

SPAIN

E-mail: casasalvero@ub.edu 